TP Periodica Polytechnica Mechanical Engineering

59(4), pp. 153-163, 2015

DOI: 10.3311/PPme.8006

Creative Commons Attribution (i)

\title{
Stall Detection Using Pseudo-Acoustic Pressure Modulation in Industrial Fans
}

\author{
Alessandro Corsini ${ }^{1,2 *}$, Sara Feudo ${ }^{1}$, Cecilia Tortora ${ }^{1}$, Graziano Ullucci $^{2}$
}

RESEARCH ARTICLE

\begin{abstract}
This work investigates the use of unconventional sensors to measure pressure modulation interpreted as pseudo sound in the near field on the casing of a fan in a view to detect rotating stall. Rotating stall is an aerodynamic issue with a frequency signature usually half the rotor frequency. In low speed turbomachines, such as industrial fans, this turns in very low frequencies, even lower than $10 \mathrm{~Hz}$.

Traditional methods use piezoelectric sensors, e.g., pressure transducers or microphones, respectively in the near and farfield, to detect instability from the signal patterns with broad frequency ranges. Recently electret microphones have been proposed, but with a cut-off frequency of $20 \mathrm{~Hz}$ as such not suitable for signal in near infrasound region.

The sensor used in this work, have a narrower frequency range than more advanced technologies. The authors developed and set-up a measurement system able to acquire low frequency pressure signals using dynamic microphones.

In this paper the authors developed a measurement chain based on dynamic microphone and pressure transducer in order to create a stall warning system. They tested the system on a low speed axial fan and they validated the work against state of the art acoustic control techniques. For this reason those devices represent candidate solutions for the detection of the patterns typical of rotating stall in turbomachines.
\end{abstract}

\section{Keywords}

DIY pressure sensors, Stall detection, Axial fan, Dynamic chaotic systems, Pattern recognition

\footnotetext{
${ }^{1}$ Department of Mechanical and Aerospace Engineering,

Sapienza Università di Roma, Via Eudossiana 18, Rome, Italy

${ }^{2}$ SED Soluzioni per l'Energia e la Diagnostica, via Colle Baiocco, Ferentino (FR), Italy

"Corresponding author, e-mail: alessandro.corsini@uniroma1.it
}

\section{Introduction}

Detection of aerodynamic instabilities, such as rotating stall, is still an open issue relevant to the design and operation of industrial turbomachines. Rotating stall and its propagation mechanisms in axial compressors and fans, widely studied in the past decades [1-6], may lead to vibrational issues, fatigue and mechanical failure of the rotor blades. Recently continuous monitoring systems, was proposed for industrial fans [7-10] to allow the early detection of the aerodynamic instabilities, so to enable a fast intervention.

The identification of aerodynamic instability phenomena and more precisely rotating stall is typically monitored using unsteady pressure or velocity measurements in the vicinity of the blade tip. Aerodynamic instabilities are detectable hydrodynamically in the near field, and acoustically since every turbomachine emits noise due to the high velocity flow passing on the rotating blades and on stationary objects in the duct, the noise intensity depends on the blades rotating velocity and on the position of those objects $[2,3]$, that means that every flow divergence from stability leads to an emitted noise variation.

Nowadays the available technologies for sensing pressure instabilities, are based on piezoelectric effect used in high frequency response pressure transducers [11], and condenser microphones $[8,12]$. The benefit in using these sensors is their high sensitivity in frequency range, spanning from few $\mathrm{Hz}$ to $20 \mathrm{kHz}$. However, in low speed fans, aerodynamic instabilities have a frequency signature typically lower than $100 \mathrm{~Hz}$, in particular rotating stall occurs at a frequency that is about half of the rotor frequency. Recently, in the attempt to develop a low-cost sensing solution, electret-based measurement microphones have been developed, exploiting the large diffusion of electret capsules in the multi-media markets. The study were limited to far-field analyses [13].

In the present work, the authors propose the use of unconventional sensors for unsteady pressure (pseudo-sound) measurements based on a commercial dynamic microphone capsule and electromagnetic induction working principle. The authors compared the signals acquired with two near-field sensors, namely the dynamic microphone and piezoresistive pressure 
a)

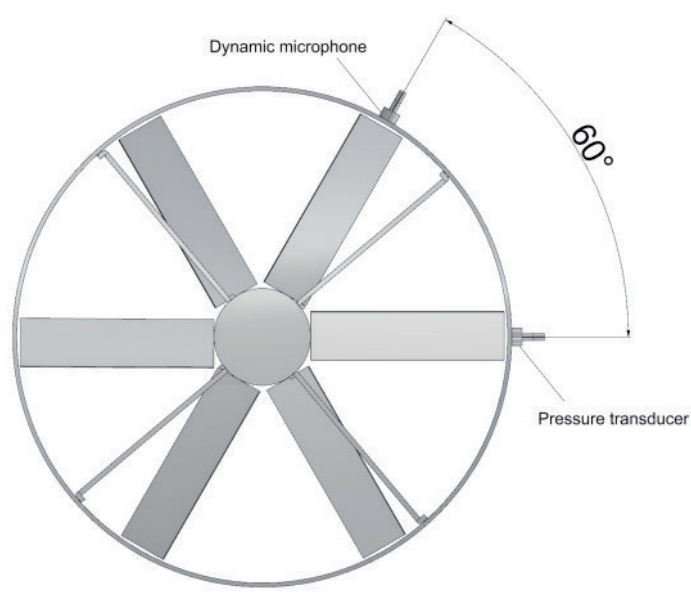

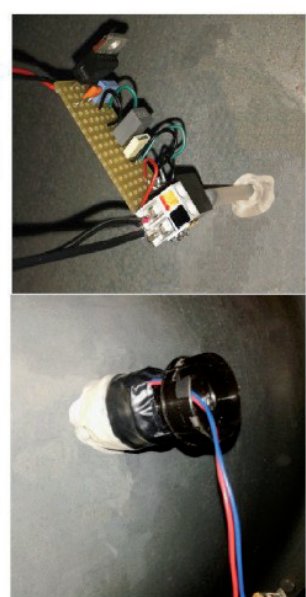

b)

c)

Fig. 1 a) position of the sensors, b) the pressure transducer, c) the microphone transducer. The processing of signals includes a spectral analysis to study the fan behaviour at stall in the frequency domain. Furthermore, the authors used the Reconstructed Phase Space (RPS) methodology and the correlated evaluation of the Lyapunov exponent to detect the dynamics and non-linearity of measured pseudo-sound signals, hidden in the time domain analysis.

\section{Nomenclature}

$\begin{array}{ll}\text { BPF } & \text { Blade Passing Frequency }[\mathrm{Hz}] \\ \mathrm{D} & \text { Embedding dimension } \\ \delta_{\mathrm{k}} & \text { Euclidean distance } \\ \text { FFT } & \text { Fast Fourier Transform } \\ \mathrm{I}(\mathrm{T}) & \text { Mutual information } \\ \mathrm{K} & \text { Kolmogorov entropy } \\ \mathrm{RI} & \text { Rotating Instability } \\ \text { RPS } & \text { Reconructed Phase Space } \\ \text { RSF } & \text { Rotating Stall Frequency } \\ s(n) & \text { Signal acquired or Scalar time series } \\ T & \text { Time delay } \\ y(n) & \text { Vector generating phase space } \\ y_{k} & \text { Reference point in phase space } \\ y^{N N} k & \text { Nearest neighbour to } y_{k} \\ \lambda_{1} & \text { Lyapunov exponent }\end{array}$

\section{Experiments}

\subsection{Fan test rig}

The turbomachine used in the experiments is a low speed industrial fan for compact cooling units. Table 1 shows the specifications for the fan.

A $1.55 \mathrm{~kW}$ direct coupled-induction 3-phase motor was used to drive the rotor at a nominal speed of $970 \mathrm{rpm}$ at open throttle. The blade passing frequency (BPF) for this operation was about $100 \mathrm{~Hz}$, and the rotor frequency $16.2 \mathrm{~Hz}$. The test rig airway is set according to the type-D configuration ISO 5801:2007 [14]. The fan was driven to stall by throttling the upstream end of the duct, 9 diameters from the fan.
Table 1 Fan data

\begin{tabular}{ll}
\hline Nominal speed & $970 \mathrm{rpm}$ \\
\hline Tip speed & $40.6 \mathrm{~m} / \mathrm{s}$ \\
Internal duct diameter & $800 \mathrm{~mm}$ \\
Blades count & 6 \\
Blades length & $200 \mathrm{~mm}$ \\
Tip clearance & $5 \mathrm{~mm}$ \\
Blade chord at the tip & $125 \mathrm{~mm}$ \\
\hline
\end{tabular}

\subsection{Instrumentation set-up}

The fan rotor casing was instrumented with two inserts, mounting a dynamic microphone and a pressure sensor respectively. The dynamic microphone in the near field was mounted with a slight recess from the inner wall of the casing since its diameter is $30 \mathrm{~mm}$ while the sensing area is only $12 \mathrm{~mm}$. The arrangement of probes is illustrated in Fig. 1.

The test sampling time interval was 60 seconds with a sample frequency of $48 \mathrm{kHz}$, set to the limit of the acquisition board. The experimental procedure was designed to throttle the upstream end of the duct, starting from a stable work condition until reaching the rotating stall with a rate of reduction of $5 \%$ of throttle per second, during 12 seconds from $0 \%$ to $60 \%$ of the throttle position, during 60 seconds of acquisition.

\section{Sensors}

\subsection{Dynamic microphone}

The microphone used in the present study is a commercial dynamic microphone. The dynamic microphone operation is based on the electromagnetic induction principle. The incoming sound pressure wave displaces a thin diaphragm, wrapped with a conductive wire coil surrounded by a magnetic field. The output is a voltage signal directly proportional to the sound 
pressure wave magnitude. The dynamic response is lower and the frequency response is less regular than ribbon or condenser microphones. Dynamic microphones are used in harsh working conditions and high noise level, due to their resistance. They are considered a solution to measure hydrodynamic pressure in the near-field of a stating equipment. Table 2 gives the microphone specifications. Figure 2 shows the microphone capsule and its design concept.

Table 2 Microphone specifications

\begin{tabular}{ll}
\hline Impedance & $600 \Omega \pm 30 \%$ \\
\hline Sensitivity & $-72 \pm 3 \mathrm{~dB}$ \\
Frequency response & $60-14 \mathrm{kHz}$ \\
\hline
\end{tabular}

Notably the lower limit of the frequency response, is not the lower frequency that the microphone is able to detect, indeed between the $60-14 \mathrm{kHz}$ the frequency response is a flat curve, for lower or higher values the curve is no longer flat, and the sound perceived is attenuated.

\subsection{Pressure transducer}

The pressure transducer used is an integrated silicon pressure sensor on-chip signal conditioned, temperature compensated and calibrated. It is a piezoresistive transducer that provides an analog output signal proportional to the measured pressure. The pressure range is -2 to $2 \mathrm{kPa}$ and the output is 0.5 to $4.5 \mathrm{~V}$. Table 3 gives the transducer specifications. Figure 3 shows the cross sectional diagram of the pressure sensor and the differential configuration on the basic chip carrier.

Table 3 Pressure transducer specifications

\begin{tabular}{ll}
\hline Typical error with auto zero & $2.5 \%$ \\
\hline Maximum error without auto zero & $6.25 \%$ \\
Sensitivity & $1.0 \mathrm{~V} / \mathrm{kPa}$ \\
Time response & $1.0 \mathrm{~ms}$ \\
Maximum pressure & $75 \mathrm{k} \mathrm{Pa}$ \\
Configuration & Differential \\
Operating compensated temperature & +10 to $+60^{\circ} \mathrm{C}$ \\
\hline
\end{tabular}
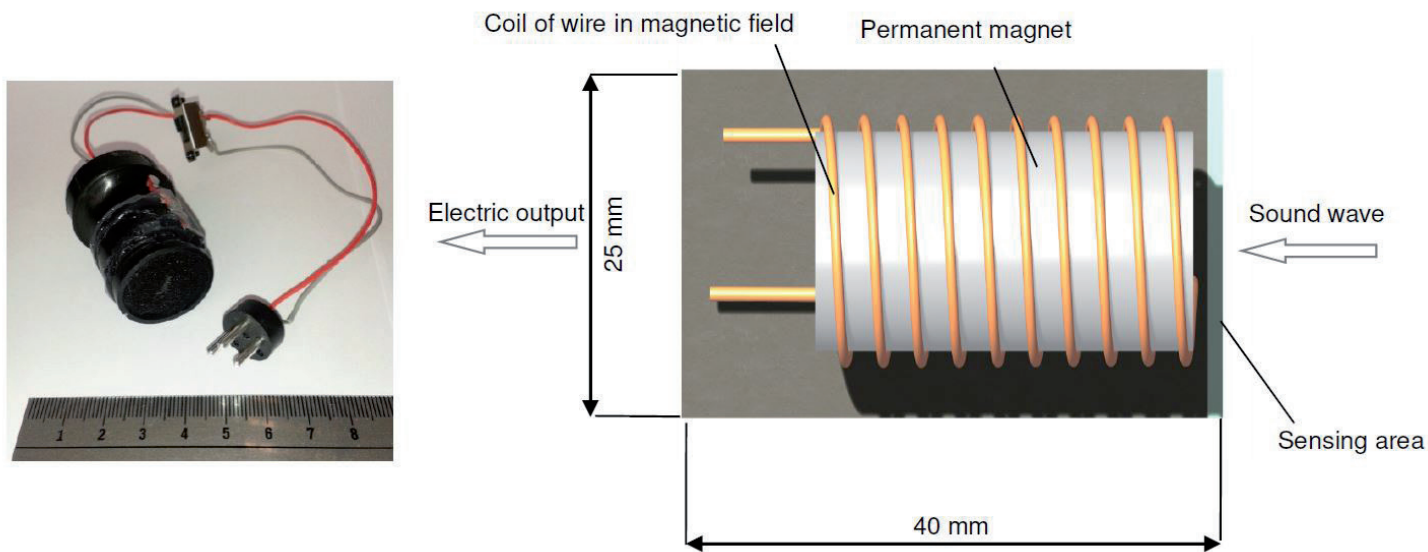

Fig. 2 The dynamic microphone
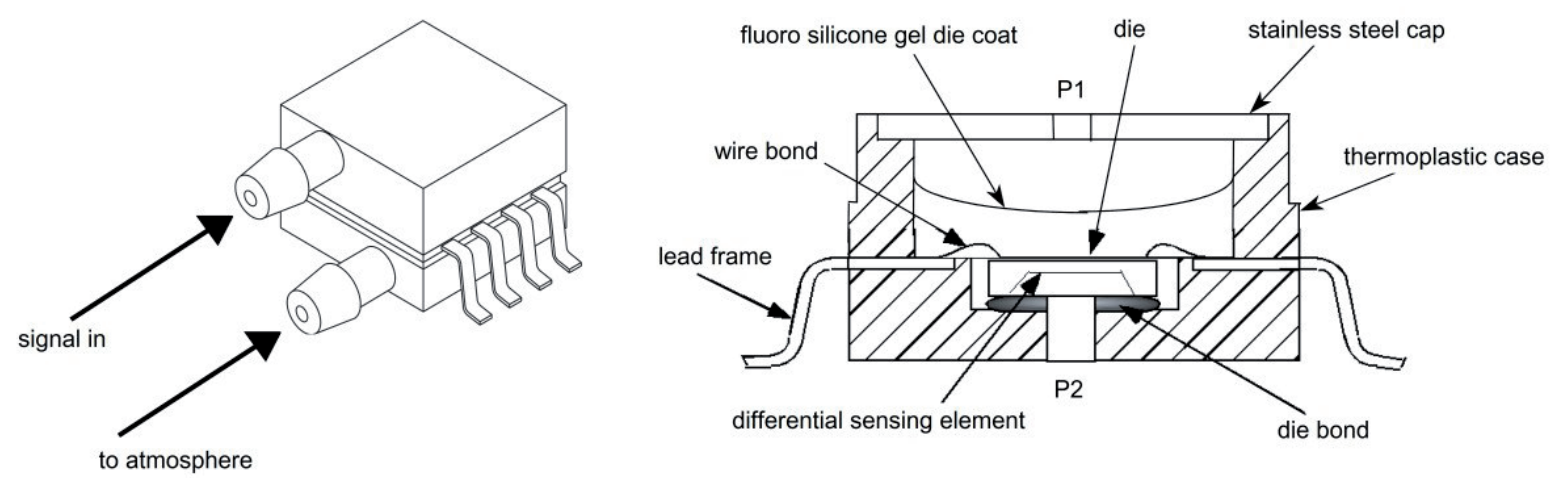

Fig. 3 Pressure sensor and its cross sectional diagram (not to scale) 


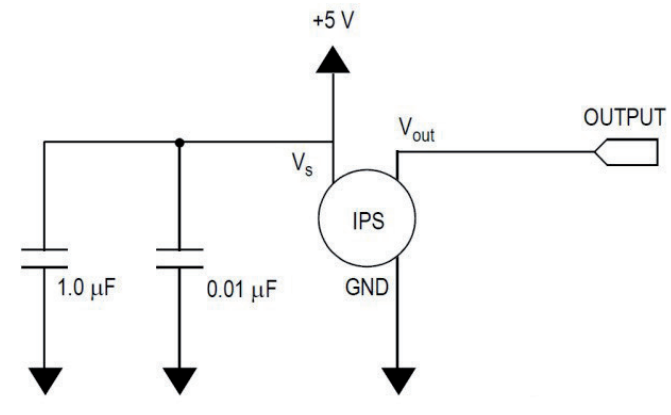

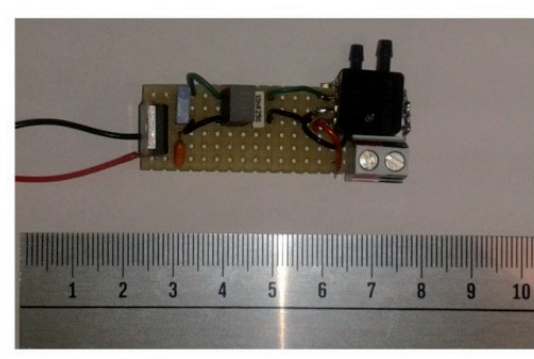

b

Fig. 4 Pressure transducer decoupling circuit: a) power supply decoupling and output filtering; b) pressure transducer final layout.

The decoupling circuit shown in Fig. 4, has been manufactured to interface the integrated sensor to the A/D input, and to comply with the transducer specifications.

\subsection{Signal amplification}

The signal from the dynamic microphone and the pressure transducer have been amplified using a DIY amplifier circuit based on a TDA2003, capable of delivering 4 Wrms at $4 \mathrm{Ohms}$. The integrated circuit is thermally and short-circuit protected. The amplifier is designed to operate with the specifications given in Table 4.

Table 4 Signal amplifier specifications

\begin{tabular}{ll}
\hline Output ower & $7 \mathrm{~W} / 4 \mathrm{Ohm}$ \\
\hline RMS output power & $3.5 \mathrm{~W} / 4 \mathrm{Ohm}$ or $2 \mathrm{~W} / 8 \mathrm{Ohm}$ \\
Total harmonic distortion & $0.05 \%(1 \mathrm{~W} / 1 \mathrm{kHz})$ \\
Frequency response & $20 \mathrm{~Hz}-20 \mathrm{kHz}(-3 \mathrm{~dB})$ \\
Input sensitivity & $40 \mathrm{mV} / 150 \mathrm{kOhm}$ \\
Signal/noise ratio & $86 \mathrm{~dB}($ A weighted $)$ \\
Power supply & $8-18 \mathrm{VDC} / 0.5 \mathrm{~A}$ \\
Dimensions & $55 \times 35 \mathrm{~mm}(2.2 ” \times 1.4 ”)$ \\
\hline
\end{tabular}

Figure 5 shows the DIY amplifier circuit and its final appearance.

\section{Measurement set-up and analysis}

The visual inspection of the time series $s(n)$ have been carried out using the dynamic microphone and the pressure sensor placed in the near field facing the flow near the blade tip. The signal acquisition lasted $60 \mathrm{~s}$ at a sample rate of $48 \mathrm{kHz}$.

The acquired signals have been low-pass filtered, with a cut-off frequency is $4.8 \mathrm{kHz}$ to highlight the low frequency response in the time-series. Figure 6 illustrates the signals acquired with the two sensors (Fig. 6a the pressure transducer, Fig. $6 \mathrm{~b}$ the dynamic microphone). In the time interval from $0 \mathrm{~s}$ to $32 \mathrm{~s}$ the authors identified the stable work condition; from 32 $\mathrm{s}$ to $45 \mathrm{~s}$ the incoming flow rate is throttled from $0 \%$ to $60 \%$, finally from $45 \mathrm{~s}$ to $60 \mathrm{~s}$ the fan was operating in stalled condition. The signal acquired with the dynamic microphone shows, during the throttling period, a remarkable sensitivity to inception of instability, that is much higher than for the piezoresistive sensor. As an example the early identification of spike-like instability at $t^{*}$. The instability is followed by an increase of the pressure fluctuation [11] as for the development of the embryonic rotating stall cell into a full stall at $t^{* *}$. This behaviour is visible, in a weaker way, also in the pressure transducer signal.

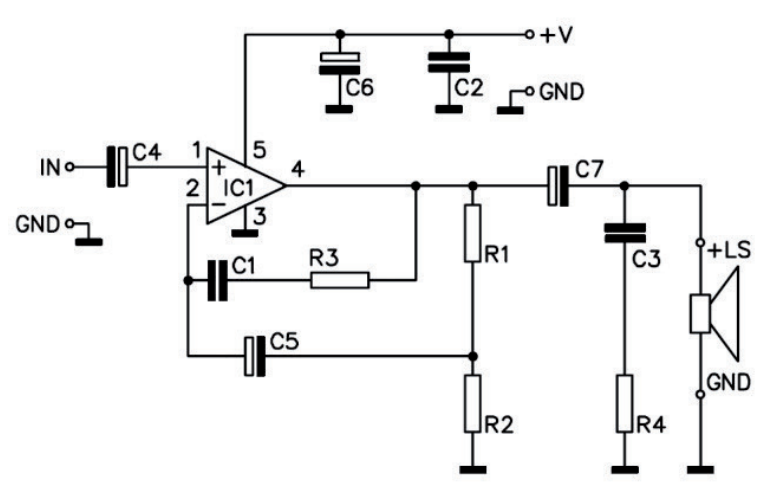

Fig. 5 The amplifier circuit

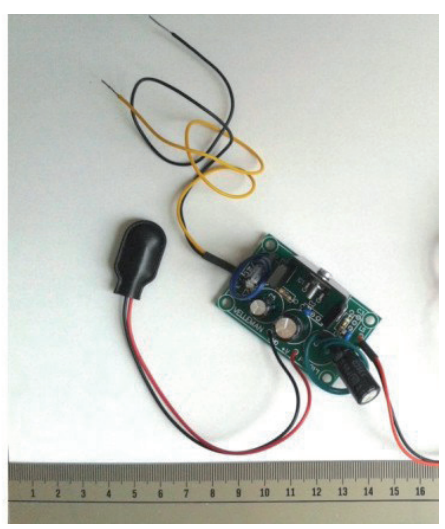

Fig. 5 The amplifier circuit 


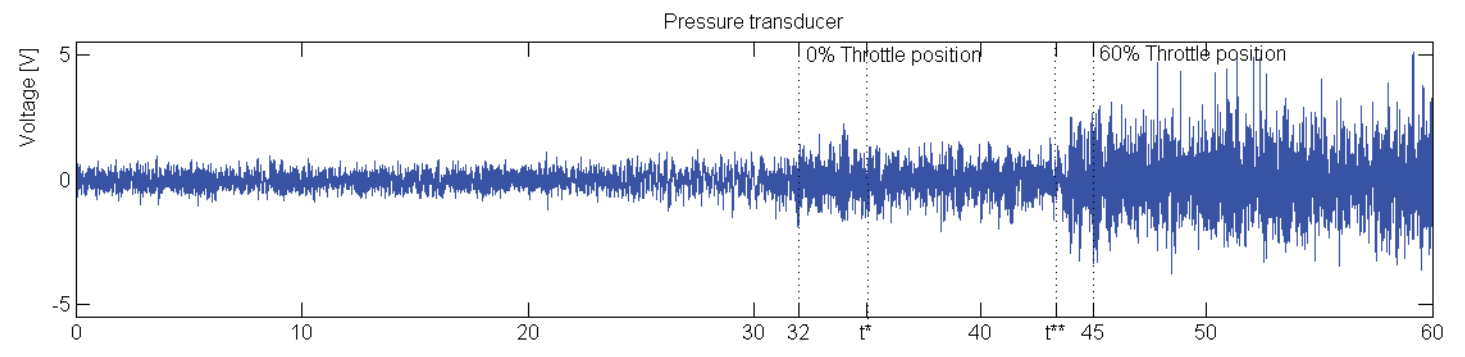

Dynamic microphone

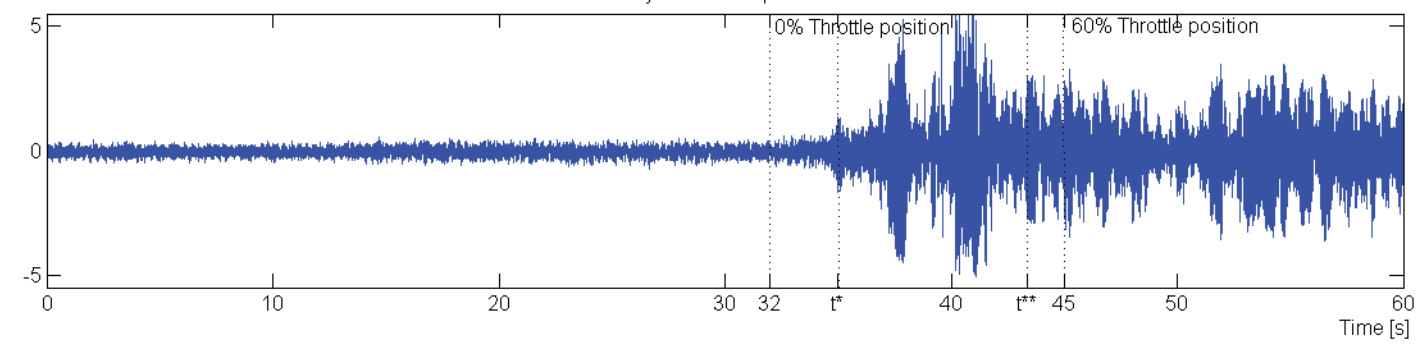

b)

Fig. 6 The acquired signals in time domain, a) pressure transducer, b) dynamic microphone

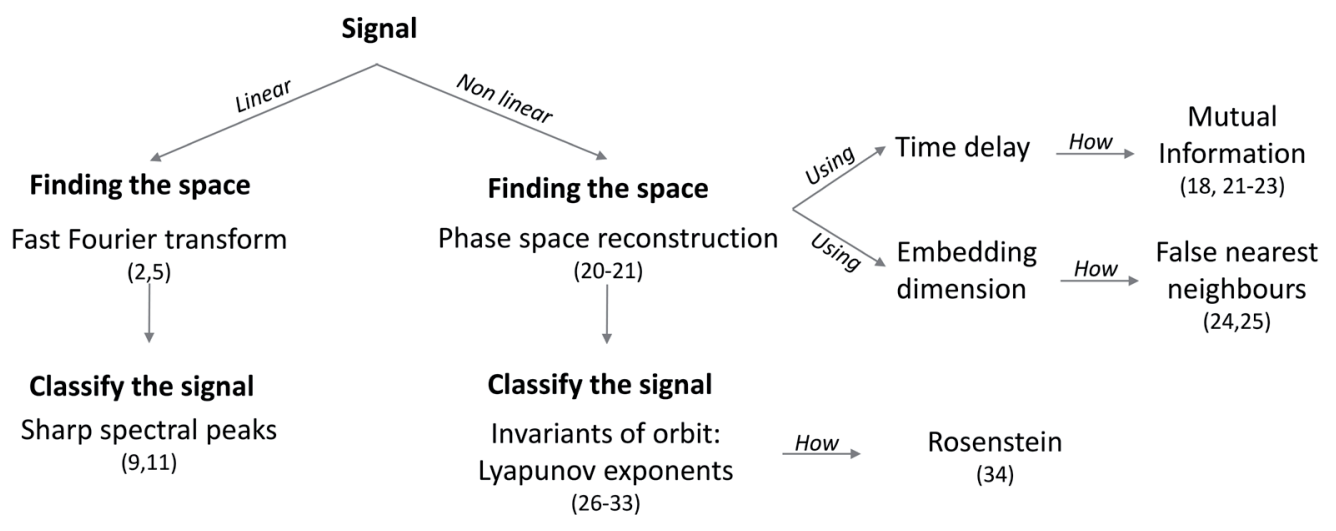

Fig. 7 Outline used for the signal analysis.

The authors applied a spectral analysis and a phase space reconstruction analysis on intervals of $1 \mathrm{~s}$ each, equivalent to 16 rotor revolutions, within the stable and the stalled operations for the sensors.

\subsection{Signal processing}

Figure 7 describes the methods used for the signal analysis, distinguishing between linear and non linear signal processing. Both methods have the same purpose, although the techniques are very different.

The spectral analysis evaluates the auto-spectrum of the measured time signal, $s(n)$ using the Fast Fourier Transform (FFT) on $1 \mathrm{~s}$ sample segments. The authors inspected the signals through the auto-spectral analysis to identify the frequency bands that reveal the rotating stall occurrence.

The auto-correlation method has been widely used for the implementation of stall and stall inception detection systems and for stability measures $[7,9,15,16]$.
In the case of a nonlinear source, it is not likely to find any simplification from Fourier-transforming the scalar measurements $s(n)$, since the processes that give rise to chaotic behavior are fundamentally multivariate. Recently, Corsini and coworkers $[8,10,17]$ have proposed stall identification based on time domain analysis and patterns reconstruction measured by "method of delays" first proposed by Takens [18]. Following this approach the authors reconstructed the phase portrait of the time series $s(n)$ by reestablishing correct space of projections. By means of RPS, as proposed by Takens [18], it is possible to identify the characteristics related to the dynamic of non-linear systems (e.g. stall), otherwise not identifiable through the usual inspection traces [18-21]. This reconstruction process allows the embedding of a data sequence, taken from the signal $s(n)$, in a $D$-dimensional space (with $D$ the embedding dimension) composed of signal segments shifted of a time lag (or time delay $T$ ).

Accordingly RPS of $s(n)$ is given in $D$ dimension by the collection of vectors $y N-(D-1) T$ and reads as: 


$$
\left[\begin{array}{l}
y_{1} \\
y_{2} \\
\vdots \\
y_{N-(D-1) T}
\end{array}\right]=\left[\begin{array}{lclc}
s_{1} & S_{1+T} & \ldots & S_{1+(D-1) T} \\
s_{2} & S_{2+T} & \ldots & s_{2+(D-1) T} \\
\vdots & \vdots & \ddots & \vdots \\
S_{N-(D-1) T} & S_{N-(D-2) T} & S_{N}
\end{array}\right]
$$

where $\left[y 1, y 2, \ldots, y_{N-(D-1) T}\right]$ are vectors generating the set of coordinates in the phase space, $\left[s 1, s 2, \ldots, s_{N-(D-1) T}\right]$ are the single information taken from the signal (i.e. the instantaneous pressure values), $D$ is the embedding dimension and $T$ is the time delay to account for the non-linearity of the signal.

Notably, to compute $T$ the authors used the First Minimum of Average Mutual Information method [18, 21-23], in which the average mutual information is kind of generalization to the nonlinear world of the correlation function in the linear world. This method provides the average amount of information after a time lag $T, s(n+T)$, obtained observing the signal $s(n)$ :

$$
\forall T, I(T)=\sum_{n-1} P(s(n), s(n+T)) \log 2\left[\frac{P(s(n), s(n+T))}{P(s(n)) P(s(n+T))}\right]
$$

here, $I(T)$ is the mutual information, $P((n))$ and $P((n+))$ are, respectively, the probability distribution of $s(n)$ and $s(n+T)$, and $P((n),(n+))$ is the joint probability distribution. Based on

the mutual information function, then, the time delay $T$ is defined as the abscissa of the first minimum of $I(T)$.

Moreover, the embedding dimension $D$ of the RPS was identified following the False Nearest Neighbors approach $[20,24,25]$. This approach, first, identifies the number of "false nearest neighbors" by comparing the magnitude of the vectors $y(n)$ in the phase portrait (i.e. points in the space of projection that appear to be nearest because of an insufficient dimension) and then selects the dimension $D$ as the one which results in zero false nearest neighbors. As such, $D$ is the dimension which unfold the portrait in the phase space.

A possible metric to compare RPS portraits could be obtained advocating the invariant analysis, in particular by introducing the Lyapunov exponents of the pattern [26-33]. Lyapunov exponents are correlated to the entropy of the signal. As proposed by Pesin [32], the sum of the positive Lyapunov exponents equals the Kolmogorov entropy $(\mathrm{K})$ or mean rate of information gain of the time series:

$$
K=\sum_{\lambda i>0} \lambda_{i}
$$

When the attractor of the RPS is chaotic, the trajectories diverge, on average. The RPS will distort, being stretched and contracted. The direction of maximum stretch of the RPS corresponds to the most unstable direction, and the largest Lyapunov exponent measures the asymptotic rate of expansion of this direction at which nearby orbits diverge. As such the presence of a positive exponent is sufficient for diagnosing chaos and represents local instability in a particular direction [33].

In this work the Lyapunov exponents were determined according to the Rosenstein approach [34], to reconstruct the attractor dynamics from a single time series $s(n)$.

$$
\delta_{k}(0)=\min _{y_{k}^{N N}}\left\|y_{k}^{N N}-y_{k}\right\|
$$

the Euclidean distance from the kth point in the RPS portrait to its neighbours; $\delta k(0)$ is assumed to diverge approximately at a rate given by the largest Lyapunov exponent $\left(\lambda_{1}\right)$ :

$$
\delta_{k}(i) \approx \delta_{k}(0) e^{\lambda_{1}(i \cdot \Delta t)}
$$

where $d_{k}(0)$ is the initial separation.

By taking the logarithm of both sides of Eq. (5), it is possible to recast the rate of change of $\delta_{k}(i)$ as:

$$
\ln \delta_{k}(i) \approx \ln C_{k}+\lambda_{1}(i \cdot \Delta t)
$$

with $i$ being the discrete-time step.

Eq. (6) represents a set of approximately parallel lines each with a slope roughly proportional to $\lambda_{1}$. The largest Lyapunov exponent is easily and accurately calculated using a leastsquares fit to the "average" line defined as

$$
y(i)=\frac{1}{\Delta t}\left\langle\ln \delta_{k}(i)\right\rangle
$$

where $\langle\ldots\rangle$ denotes the average over all values of $i$. This process of averaging is the key to calculate accurate values of $\lambda_{1}$ using small, noisy data sets [34].

\section{Results}

\subsection{Spectral analysis}

Figure 8 compares the auto-spectra of the signal measured by the pressure transducer and the dynamic microphone during the stable condition, i.e. between 0 and 30 s. The auto-spectra shows a comparable behavior for the frequency range above the first harmonic of the BPF, while in the frequency range of the rotating instability under scrutiny, i.e. which is in the order of the RF (rotating frequency), it is evident how the pressure transducer features have a higher response than the dynamic microphone.

In this respect, even the peak at RF is not well identified by the magnetic induction probe.

Figure 9, then, illustrates and compares the auto-spectra of the signals measured by the pressure transducer and microphone in stalled operation.

The major frequency signatures at BPF and RF appeared to be well captured by both the probes, which gave back even a small slippage of the motor, e.g. $100 \mathrm{~Hz}$ to $97 \mathrm{~Hz}$ at BPF and $16 \mathrm{~Hz}$ to 


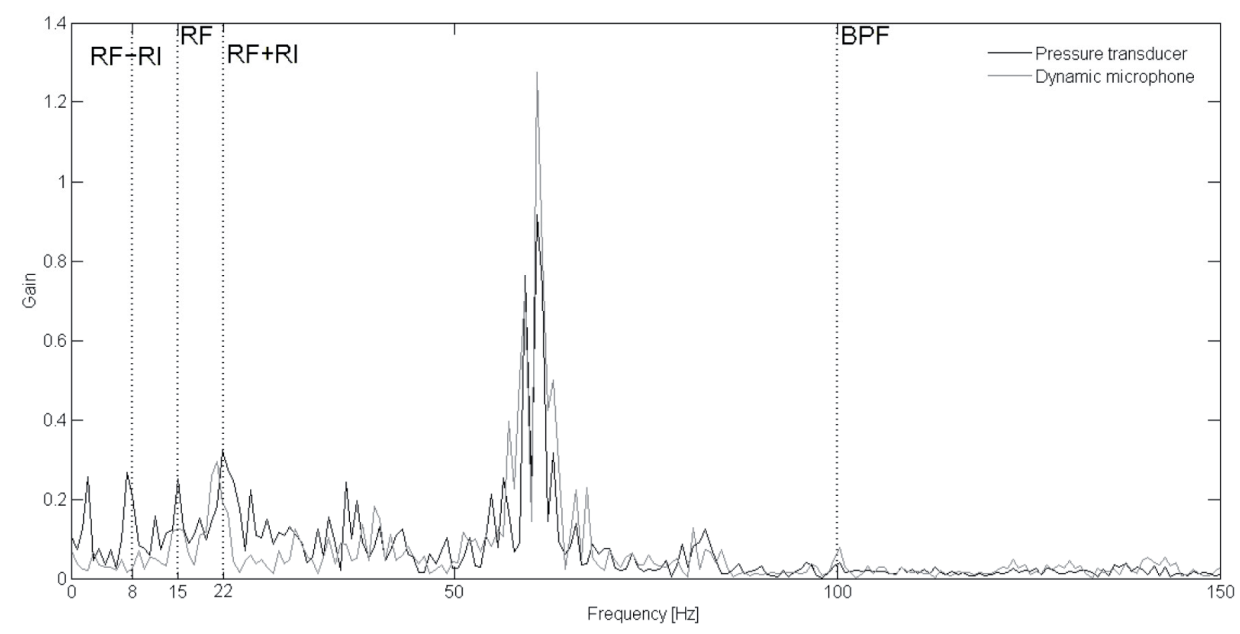

Fig. 8 Stable condition spectra

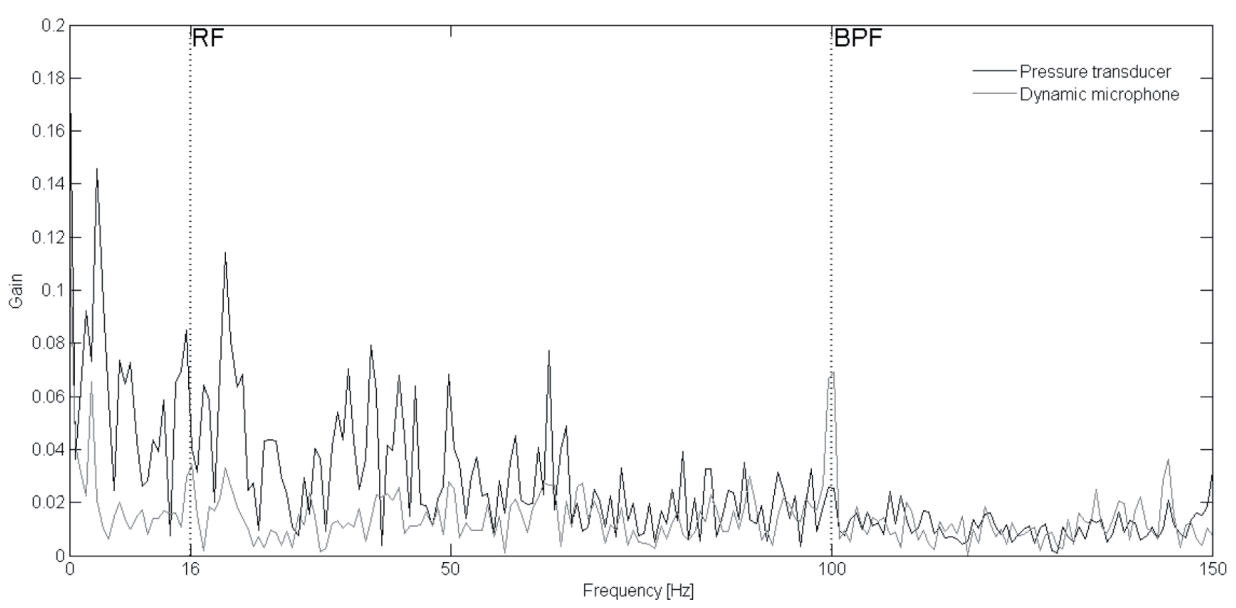

Fig. 9 Stalled condition spectra

$15 \mathrm{~Hz}$ at RF, due to the increased power absorption at stall. Below this frequency threshold only the piezoresistive probe is able to measure the peak at about $50 \%$ of RF that could be linked with the presence of a rotating stall. To further support this finding, it is worth noting that the rotating stall frequency (or rotating instability: RI) could be inferred by the evidence of two side-bands of the RF whose with a frequency pitch of $8 \mathrm{~Hz}$, i.e. that corresponds to the RSF. To this end, the dynamic microphone unable to sense the RSF directly gives the evidence of the rotor frequency as well as that of the higher RF side-band at RF+RSF. Concerning the peak at $64 \mathrm{~Hz}$ it is interpreted as a resonance frequency due to the rotorstruts interaction, in the stalled condition.

\subsection{RPS analysis}

Figure 10 shows the RPS derived from the pressure signals at stable operating condition acquired with the pressure transducer (Fig. 10a) and the dynamic microphone (Fig. 10b). The RPS are reconstructed using an embedding dimension $D=2$, while the time lag $T$ is respectively equal to 5 for the pressure transducer and 4 for the dynamic microphone.

Figure 11, on the other hand, RPS derived from the pressure signals at stall acquired with the pressure transducer (Fig. 11a) and the dynamic microphone (Fig. 11b). The RPS are reconstructed using an embedding dimension $D=2$, while the time lag $T$ is respectively equal to 7 for the pressure transducer and 8 for the dynamic microphone.

In contrast with the evidence of the spectral analysis, the portrait comparison indicates that both the signals from the pressure transducer and the dynamic microphone are able to create phase-space patterns which identify sharply the evolution from stable to stalled operations. The signature of such evolution being the diagonal stretching of patterns and, as already explained, this circumstance could be correlated with the predominance, at stall, of a chaotic behavior with the orbits of RPS attractor evolving in a complex shape; whereby some directions are contracted and other are stretched.

Comparing Fig. 10 and 11 it is possible to recognize two well defined directions of stretching/contraction. In particular the stretching direction corresponds to the unstable direction that is proportional to the maximum Lyapunov exponent. Unlike the spectral analysis, in the RPS both the sensors show almost the same response.

To further support the portrait comparison, Fig. 12 shows the evolution in time of the maximum Lyapunov exponent 

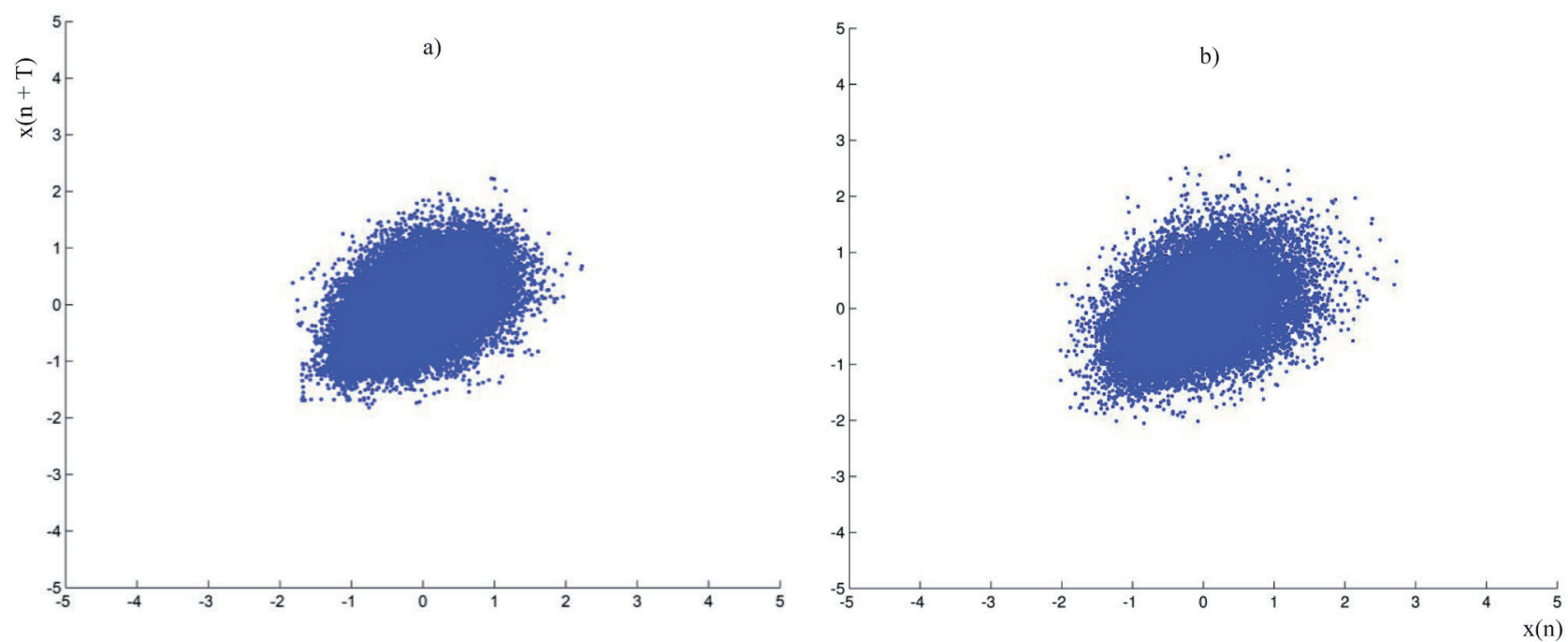

Fig. 10 RPS at stable operations for a) the pressure transducer, and b) the dynamic microphone
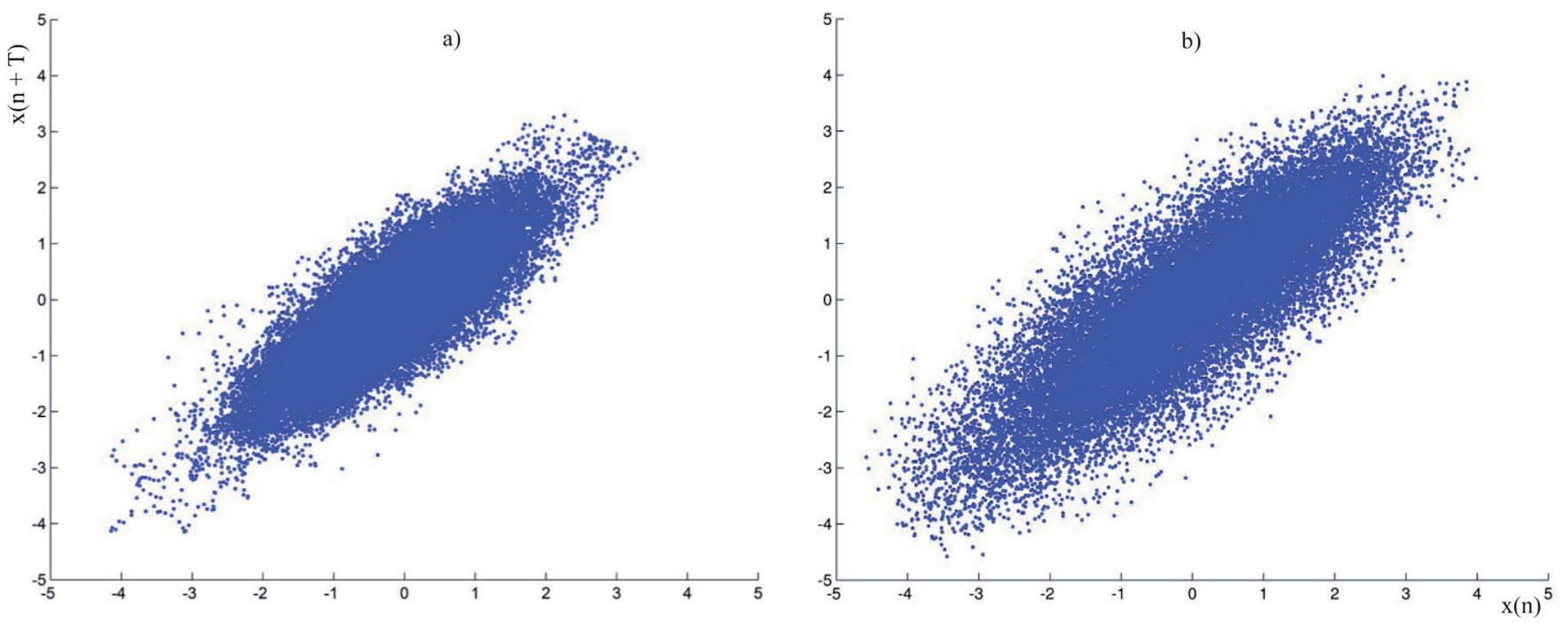

Fig. 11 RPS at stalled operations for a) the pressure transducer, and b) the dynamic microphone

relative to the RPS. The Lyapunov exponent was computed on data samples starting from a stable operating condition towards the aerodynamic instability for the signal acquired with the pressure transducer (Fig. 12.a) and dynamic microphone (Fig. 12.b).

The vertical lines identify, respectively, the stable operating interval (0-32 s), the throttling transient $0 \%$ to $60 \%(32-45 \mathrm{~s})$, and stalled condition (45-60 s).

Both the pressure transducer and the dynamic microphone RPS, featured a Lyapunov exponent time evolution able to detect the change in operating conditions and, which is of higher importance to condition monitoring, the incipience of rotating instabilities during the throttling transient with Lyapunov exponents which increased evidently before the proper stall. As already explained, the Lyapunov exponent metric represents a local instability and the presence of a positive exponent is sufficient for diagnosing chaos. Therefore, the recorded time evolutions of Lyapunov exponents infer the chaotic nature of pressure signals at stall. Moreover, since the sum of the positive Lyapunov exponents, equals the Kolmogorov entropy, it is possible to confirm that in stalled operation the system increases its entropy.

Table 5 Lyapunov statistics during stable and stalled condition

\begin{tabular}{lllll}
\hline Sensor & Operation & Mean & Min & Max \\
\hline \multirow{2}{*}{ Pressure transducer } & Stable & 0.78 & -2.17 & 5.81 \\
& Stalled & 4.78 & -0.61 & 12.34 \\
& Stable & 1.97 & 0.36 & 4.70 \\
Dynamic microphone & Stalled & 4.07 & 0.46 & 9.36 \\
\hline
\end{tabular}

Table 5 illustrates the statistics of the Lyapunov exponent variation in stable and stalled condition for both the probes. 

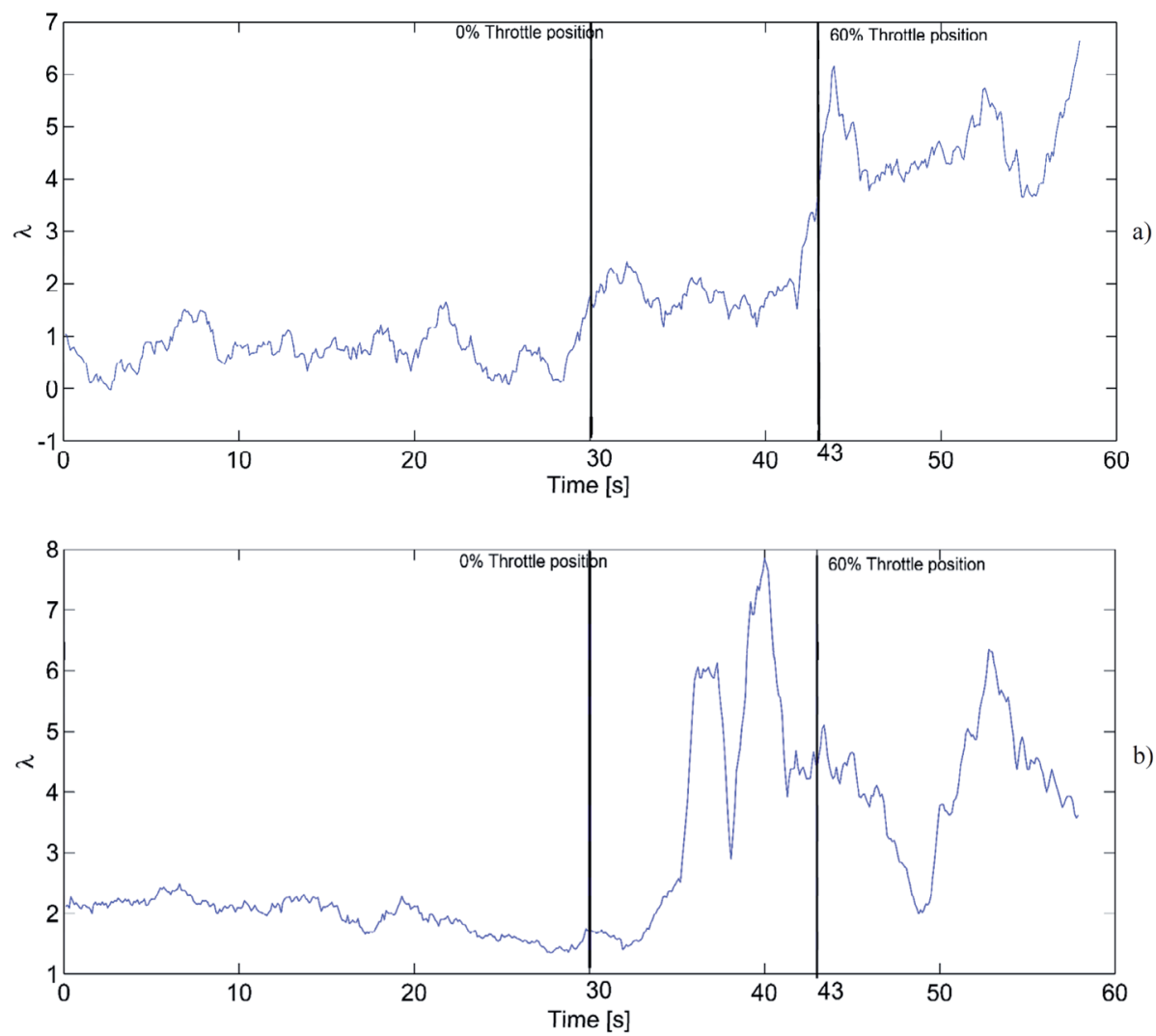

Fig. 12 Evolution in time of the Lyapunov exponent a) pressure transducer, and b) dynamic microphone
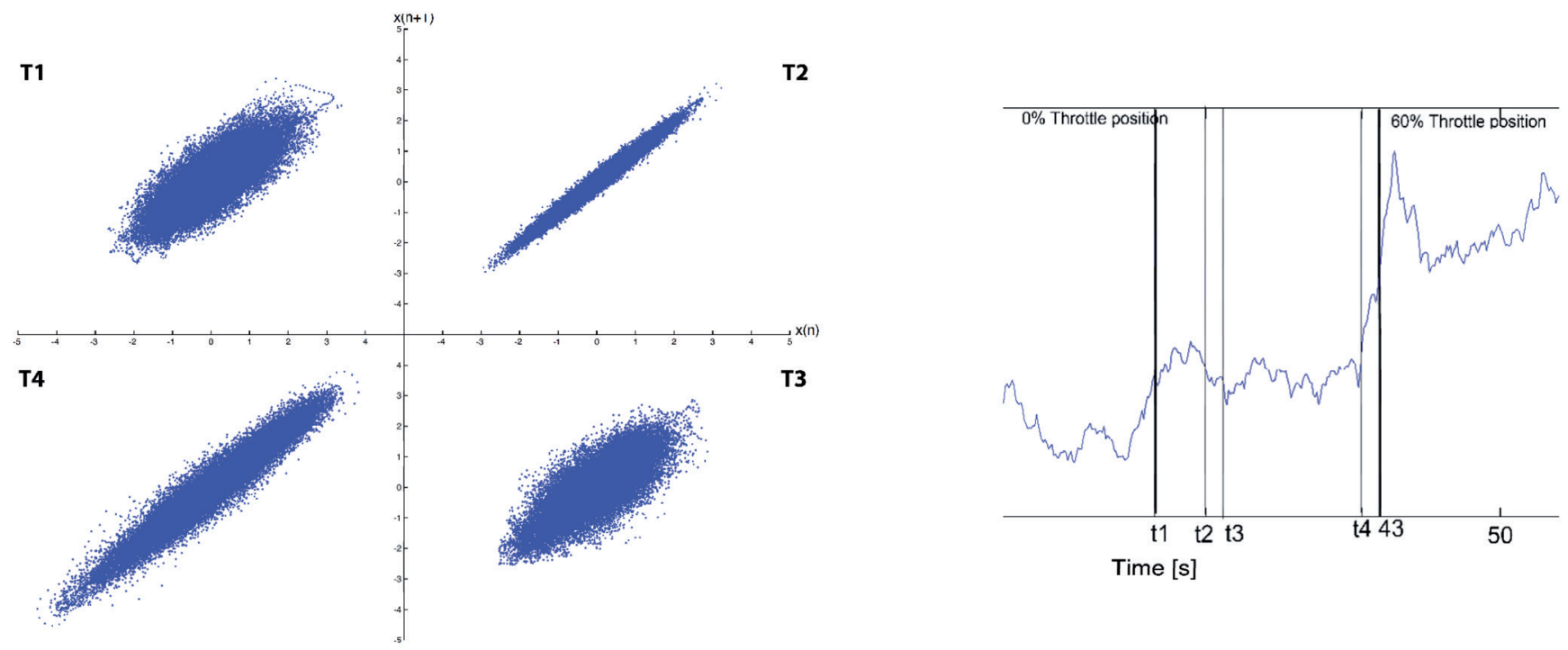

Fig. 13 Throttling condition for the pressure transducer

Once more, the comparison of the data in Table 5 confirms that contrarily to the Fourier analysis the dynamic microphone performs in a similar way to the piezoresistive probe the only limit being the retarded response in the rise of the Lyapunov exponent.

As demonstrated by the evolution in time (Fig. 12 shows) the instability of the Lyapunov exponent begins already in the throttling interval. For this reason, to provide additional hints on this transient, Fig. 13 and 14 combine the time evolution of Lyapunov exponents to the RPS portraits for different instants during the throttling interval. Figure 13 and Figure 14 illustrate the behaviour of the pressure history measured with the pressure transducers and the dynamic microphone, respectively.

Four time instants during the throttling transient have been considered, namely $\mathrm{t} 1=32 \mathrm{~s}, \mathrm{t} 2=35 \mathrm{~s}, \mathrm{t} 3=36 \mathrm{~s}$, and $\mathrm{t} 4=44$ $\mathrm{s}$ for the pressure traducer (Fig. 13) and $\mathrm{t} 1=32 \mathrm{~s}, \mathrm{t} 2=36 \mathrm{~s}$, $\mathrm{t} 3=41 \mathrm{~s}$, and $\mathrm{t} 4=44 \mathrm{~s}$ for the dynamic microphone (Fig. 14). The RPS portraits have been computed using an embedding dimension $\mathrm{D}=2$, and time lag $\mathrm{T}$ in the range 1 to 15 . 


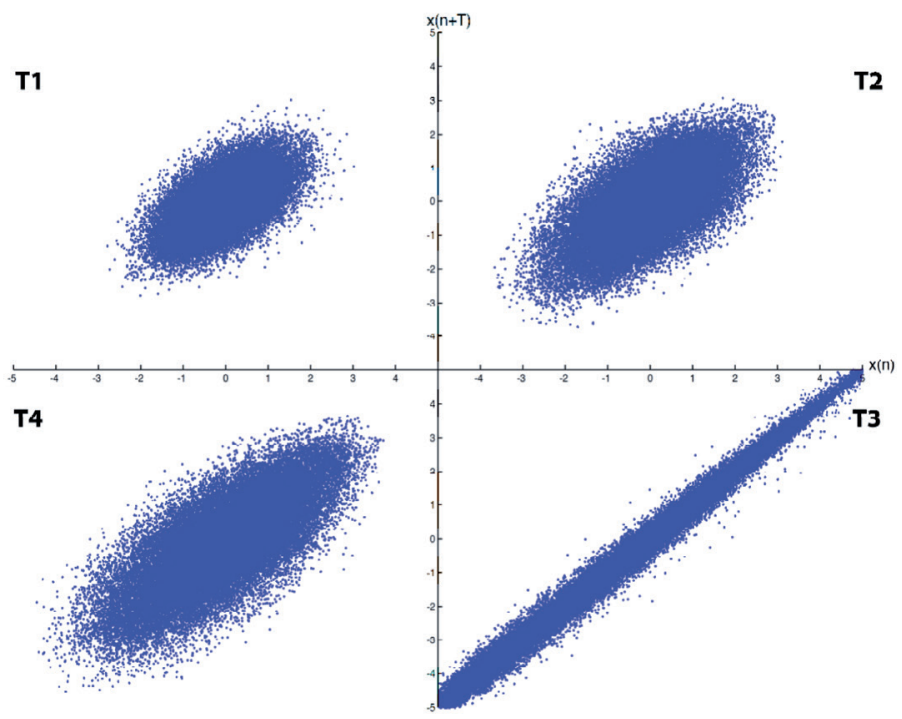

$\mathbf{T}$

Fig. 14 Throttling condition for the dynamic microphone

The observation of Fig. 13 and 14 permits to detect another interesting feature of the chaotic behaviouur of the pressure signals during stall incipience which is not detectable by the analysis of the Lyapunov exponent analysis.

Remarkably, the patterns feature a periodic stretching and folding of the attractor as for the pulsation of the phase-space portraits. This information complement the Lyapunov exponent analysis, which only measures the maximum exponent corresponding to the stretching direction and neglects the other direction. In this respect, both the probing technologies recognize the non-linear dynamic of the pressure signals when stall conditions established.

\section{Conclusion}

From the frequency domain analysis through the Fast Fourier Transform is noticeable that rotating stall occurs at low frequencies, lower than $100 \mathrm{~Hz}$; more importantly the rotating stall is visible with both the instruments. The clearly visible frequency peak at $64 \mathrm{~Hz}$ during stalled operations is considered to be a resonant frequency due to the rotor-struts interaction. The experiment proves that the dynamic microphone is able to detect the presence of aerodynamic instabilities at frequencies lower than $20 \mathrm{~Hz}$ with a 50 $\div 60 \%$ precision. Indeed applying the Spectral Analysis on the dynamic microphone signal, it is possible to detect a peak at about $15 \mathrm{~Hz}$ which is near the value $16.2 \mathrm{~Hz}$ of the rotor frequency.

Rotating stall is a non-linear phenomenon, for this reason it is not possible to analyse it with in frequency domain analysis. It is necessary to employ methodology ad hoc for non linear system analysis as those related to chaotic systems.

Using the RPS method the authors identified the rotating stall typical pattern. As in the Spectral Analysis, the method response is more chaotic, and the pattern tends to enlarge and extend in a determined direction, corresponding to instable direction, while approaching the unsteady condition.
This assertion is further confirmed by the Lyapunov spectrum that shows the increasing of chaoticness, therefore of the entropy of the system, during stalled condition.

Both the used methods have the same purpose and although the techniques are very different, they supply the same results but with a different rate of noticeability.

Both the pressure transducer and the dynamic microphone have detected the presence of the rotating stall phenomenon, and the analysis methods used confirmed the validity of the used instruments.

\section{References}

[1] Bianchi, S., Corsini, A., Sheard, A. G., Tortora, C. "A Critical Review of Stall Control Techniques in Industrial Fans." ISRN Mechanical Engineering. 2013. pp. 1-18. 2013. DOI: 10.1155/2013/526192

[2] Cumpsty, N. A. "A critical review of turbomachinery noise." Journal of Fluids Engineering. 99 (2). pp. 278-293. 1977. DOI: 10.1115/1.3448745

[3] Cumpsty, N. A. "Sum and difference tones from turbomachines." Journal of Sound and Vibration. 32 (3). pp. 383-386. 1974.

DOI: $10.1016 / \mathrm{s} 0022-460 x(74) 80094-5$

[4] Greitzer, E. M. "Surge and Rotating Stall in Axial Flow Compressors Part I: Theoretical Compression System Model." Journal of Enginering for Power. 98 (2). pp. 190-198. 1976. DOI: 10.1115/1.3446138

[5] Day, I. J. "Active Suppression of Rotating Stall and Surge in Axial Compressors." Journal of Turbomachinery. 115 (1). pp. 40-47. 1993 DOI: $10.1115 / 1.2929216$

[6] Paduano, J. D., Epstein, A. H., Valavani, L., Longley, J. P., Greitzer, E. M., Guenette, G. R. "Active Control of Rotating Stall in a Low-Speed Axial Compressor." Journal of Turbomachinery. 115 (1). pp. 48-56. 1993. DOI: $10.1115 / 1.2929217$

[7] Tahara, N., Kurosaki, M., Ohta, Y., Outa, E., Nakajima, T., Nakakita, T. "Early Stall Warning Technique for Axial-Flow Compressors." Journal of Turbomachinery. 129 (3). pp. 448-456. 2006. DOI: 10.1115/1.2447948

[8] Bianchi, S., Corsini, A., Sheard, A. G. "Detection of stall regions in a low-speed axial fan. Part i - azimuthal acoustic measurements." Proceedings of ASME Turbo Expo: Controls, Diagnostics and Instrumentation; Cycle Innovations; Marine. 2010. DOI: 10.1115/gt2010-22753 
[9] Tahara, N., Nakajima, T., Kurosaki, M., Ohta, Y., Outa, E., Nishikawa, T. "Active stall control with practicable stall prediction system using auto-correlation coefficient." In: 37th Joint Propulsion Conference and Exhibit. Salt Lake City. 2001 DOI: 10.2514/6.2001-3623

[10] Sheard, A.G., Corsini, A., Bianchi, S. "Stall Warning in Low Speed Axial Fan by Visualization of Sound Signal." Journal of Engineering for Gas Turbines and Power. 133 (4). pp. 041601-041601-10. 2011. DOI: $10.1115 / 1.4002178$

[11] Bianchi, S., Corsini, A., Mazzucco, L., Monteleone, L., Sheard, A. G. "Stall Inception, Evolution and Control in a Low Speed Axial Fan With Variable Pitch in Motion." Journal of Engineering for Gas Turbines and Power. 134 (4). pp. 042602. DOI: 10.1115/1.4004726

[12] Zhu, T., Carolus, T. H. "Experimental and unsteady numerical investigation of the tip clearance noise of an axial fan." In: ASME 2013 Turbine Blade Tip Symposium. Hamburg, Germany, 30 September-3 October 2013. DOI: $10.1115 /$ tbts2013-2034

[13] Bennett, G. J., Mahon, J., Hunt, S., Harris, C. "Design of an Electret Based Measurement Microphone." In: 26th International Manufacturing Conference (IMC26). pp. 411-418. 2009.

[14] ISO 5801. Industrial Fans-Performance Testing Using Standardized Airways. 2007.

[15] Dhingra, M., Neumeier, Y., Prasad, J. V. R., Breeze-Stringfellow, A., Shin, H.-W., Szucs, P. N. "A Stochastic Model for a Compressor Stability Measure." In: ASME Turbo Expo 2006: Power for Land, Sea, and Air. Volume 2. Barcelona, Spain, May 8-11, 2006. DOI: 10.1115/gt2006-91182

[16] Christensen, D., Cantin, P., Gutz, D., Szucs, P. N., Wadia, A. R., Armor, J., Dhingra, M., Neumeier, Y., Prasad, J. V. R. "Development and Demonstration of a Stability Management System for Gas Turbine Engines." In: ASME Turbo Expo 2006: Power for Land, Sea, and Air. Vol. 6. Turbomachinery, Parts A and B, Barcelona, Spain, May 8-11, 2006. 2006. DOI: $10.1115 / \mathrm{gt} 2006-90324$

[17] Bianchi, S., Corsini, A., Sheard, A. G. "Demonstration of a stall detection system for induced-draft fans." Journal of Power \& Energy. 2013. DOI: $10.1177 / 0957650912475146$

[18] Takens, F. "Detecting strange attractors in turbulence." In: Rand, D., Young, L.-S. (ed.) Dynamical Systems and Turbulence, Warwick 1980., Lecture Notes in Mathematics. 898. pp. 366-381. 1981. DOI: $10.1007 / \mathrm{Bfb} 0091924$

[19] Manè, R. "On the dimension of the compact invariant sets of certain nonlinear maps." In: Rand, D., Young, L.-S. (ed.) Dynamical Systems and Turbulence, Warwick 1980., Lecture Notes in Mathematics. Vol. 898. pp. 230-242. 1981. DOI: 10.1007/Bfb0091916

[20] Abarbanel, H. D. I., Brown, R., Sidorowich, J. J., Tsimring, L. S. "The analysis of observed data in physical systems." Reviews of Modern Physics. 65 (4). pp. 1343-1351. 1993. DOI: 10.1103/revmodphys.65.1331
[21] Packard, N. H., Crutchfield, J. P., Farmer, J. D., Shaw, R. S. "Geometry from a time series." Physical Review Letters. 45 (9). pp. 712-716. 1980. DOI: $10.1103 /$ physrevlett. 45.712

[22] Gallager, R."Information Theory and Reliable Communication." 1968. DOI: 10.1007/978-3-7091-2945-6

[23] Abarbanel, H. D. I., Masuda, N., Rabinovich, M. I., Tumer, E. "Distribution of mutual information." Physics Letters A. 281 (5-6). pp. 368-373. 2001. DOI: 10.1016/S0375-9601(01)00128-1

[24] Kennel, M. B., Brown, R., Abarbanel, H. D. I. "Determining embedding dimension for phase space reconstruction using a geometrical construction." Physical Review A. 45 (6). pp. 3403-3411. 1992.

DOI: 10.1103 /physreva.45.3403

[25] Hegger, R., Kantz, H. "Improved false nearest neighbor method to detect determinism in time series data." Physical Review E. 60 (4). pp. 49704973. DOI: 10.1103 /physreve. 60.4970

[26] Wright, J. "Method for Calculating a Lyapunov exponent." Physical Review A. 29 (5). pp. 2924-2927. 1984. DOI: 10.1103/physreva.29.2924

[27] Sato, S., Sano, M., Sawada, Y. "Practical Methods of Measuring the Generalized Dimension and Largest Lyapunov Exponents in High-Dimensional Chaotic Systems." Progress of Theoretical Physics. 77 (1). pp. 1-5. 1987. DOI: 10.1143/PTP.77.1

[28] Goldhirsch, I., Sulem, P. L., Orszag, S. A. "Stability and Lyapunov Stability of Dynamical Systems: a Differential Approach and Numerical Method." Physica D: Nonlinear Phenomena. 27 (3). pp. 311-337. 1986. DOI: 10.1016/0167-2789(87)90034-0

[29] Geist, K., Parlitz, U., Lauterborn, W. "Comparison of Different Methods for Computing Lyapunov Exponents." Progress of Theoretical Physics. 83 (5). pp. 875-893. 1990. DOI: 10.1143/PTP.83.875

[30] Abarbanel, H. D. I., Brown, R., Kennel, M. B. "Lyapunov Exponents in Chaotic Systems: Their Importance and Their Evaluation using Observed Data." International Journal of Modern Physics B. 5 (09). pp. 1347-1375. 1991. DOI: 10.1142/s021797929100064x

[31] Zeng, X., Eykholt, R., Pielke, R. A. "Estimating the Lyapunov Exponent Spectrum from Short Time Series of Low Precision." Physical Review Letters. 66 (25). pp. 3229-3232. 1991. DOI: 10.1103/physrevlett.66.3229

[32] Pesin, Y. B. "Characteristic Lyapunov Exponents and Smooth Ergodic Theory." Russian Mathematical Surveys. 32 (4). pp. 55-114. 1977. DOI: 10.1070/rm1977v032n04abeh001639

[33] Wolf, A., Swift, J. B., Swinney, H. L., Vastano, J. A. "Determining Lyapunov exponents from a time series." Physica D: Nonlinear Phenomena. 16 (3). pp. 285-317. 1985. DOI: 10.1016/0167-2789(85)90011-9

[34] Rosenstein, M. T., Collins, J. J., De Luca, C. J. "A practical method for calculating largest Lyapunov exponents from small data sets." Physica D: Nonlinear Phenomena. 65 (1-2). pp. 117-134. 1993. DOI: 10.1016/0167-2789(93)90009-p 